\title{
Unstable Inverse Heat Transfer Problems in Microelectronics
}

\author{
G. De $\mathrm{MeY}^{a, *}$, B. Boguseawski ${ }^{b}$ AND A. KOS ${ }^{b}$ \\ ${ }^{a}$ University of Ghent, Department of Electronics and Information Systems \\ Sint Pietersnieuwstraat 41, 9000 Gent, Belgium \\ ${ }^{b} \mathrm{AGH}$ University of Science and Technology, Institute of Electronics \\ al. A. Mickiewicza 30, 30-059 Kraków, Poland
}

(Received August 6, 2012; in final form January 15, 2013)

\begin{abstract}
Inverse heat transfer problems are very important for the thermal testability of integrated circuits. Temperature sensors integrated on the same chip measure in real time the power dissipation in one or more critical heat sources of the circuit in order to prevent overheating. It will be demonstrated that these kinds of problems can give rise to mathematical unstabilities or the ill conditioning of the inverse problem. This statement will be proved with the help of several particular cases.
\end{abstract}

DOI: $10.12693 /$ APhysPolA.123.637

PACS: $02.30 . \mathrm{zz}, 44.05 .+\mathrm{e}, 85.40 . \mathrm{Qx}$

\section{Introduction}

Thermal problems in electronics have gained a lot of attention in recent years [1]. The main reason is that both the power and the power density increased considerably. In order to guarantee a sufficient lifetime, the maximum temperature should be kept below a certain limit [2]. But even when the maximum temperature is acceptable, the temperature distribution on a silicon chip can cause malfunctioning of the circuit. Taking into account the temperature dependence of semiconductor characteristics, the behaviour of a circuit or a component will also change due to the temperature distribution in the silicon $[3,4]$.

Usually, the solution of thermal problems involves the determination of the temperature distribution starting from the known power dissipations in the individual components. In this contribution the inverse thermal problem will be considered: determine the power density starting from temperature measurements on the chip. Numerically, these kinds of problems are known as "inverse" problems and are often ill conditioned, i.e. small errors of the temperature measurements cause large errors on the calculated powers.

The inverse heat transfer problem in electronics and microelectronics has been the topic of several papers [5-9]. Most of these papers deal with the numerical problem associated with the ill conditioning of the system matrix [10]. An overview of numerical techniques to invert ill conditioned matrices can be found in [11].

Inverse thermal problems in microelectronics become more and more important. Along the boundary of an integrated circuit, one can integrate temperature sensors, so that the power dissipation can be monitored. If nec-

*corresponding author; e-mail: demey@elis.ugent.be essary, a circuit interrupt or a decrease of the clock frequency can be foreseen in order to prevent excessive heating. This approach is called design for testability, i.e. the design is made in such a way that the circuit can also be tested during normal operation [12]. Not only the original circuit but also a temperature sensing circuit is integrated on the same chip. These temperature sensors cannot be put on the same place as the power sources. It will be demonstrated that the finite distance between sensors and sources or more generally the layout is responsible for the ill conditioned behaviour of the inverse problem.

In this contribution we will limit ourselves to a few cases: (1) two heat sources and two sensors, (2) two heat sources and three sensors, (3) three heat sources and five sensors and (4) two heat sources and 32 sensors along the chip boundary. At the end an exact mathematical analysis will be provided for the simplest case in order to explain the unexpected results found with the numerical simulations.

\section{Model for the temperature distribution}

We assume that the integrated circuit can be (thermally) approximated by a half infinite space with thermal conductivity $k$. In the vicinity of a heat source, the heat is only transported by conduction. The temperature $T$ in a sensing point is then given by [13]:

$$
T=\frac{P}{2 \pi k r},
$$

where $P$ is the dissipated power and $r$ the distance between the sensing point and the heat source.

If several heat sources are involved, superposition can be used

$$
T=\sum_{i} \frac{P_{i}}{2 \pi k r_{i}} .
$$

$r_{i}$ denotes the distance between the sensing point and the $i$-th heat source $P_{i}$. 
One may argue that the thermal model as expressed by (2) is a very simplified one. The model does not take into account the influence of the package, the cooling fins, the convective cooling,... It must be remarked here that our model only needs to take into account the spatial temperature variations on the chip, and not the average heating. The package, cooling fin and convection will just yield an average temperature rise of the whole chip. For a more realistic temperature distribution, this average temperature has to be superposed to the value given by (2). In this paper we are only interested in the spatial temperature variations on the chip surface, for which (2) is a good model also used by other authors [14].

The expressions (1) and (2) implicitly assume that every heat source is a point shaped one. In practice it means that (1) or (2) can only be used at a certain distance $r$ from the heat source. It was found that the approximations (1) and (2) are valid for distances less than or comparable to the thickness of the silicon substrate. For very thin substrates $(25 \mu \mathrm{m})$ the present model is not suitable.

\section{Two heat sources}

Assume two power sources $P_{1}$ and $P_{2}$ located on the $x$-axis (Fig. 1). The first sensing element $S_{1}$ is placed

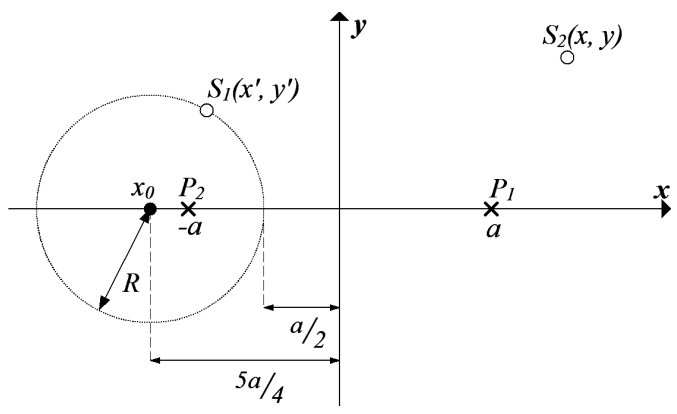

Fig. 1. Two heat sources $P_{1}$ and $P_{2}$ and two sensors $S_{1}$ and $S_{2}$ : the circle of Appolonius.

in the point $\left(x^{\prime}, y^{\prime}\right)$. The question now is in which point $(x, y)$ the second sensor $S_{2}$ should not be placed in order to guarantee the solution of the inverse problem, i.e. the determination of $P_{1}$ and $P_{2}$ from the measured temperatures $T_{1}$ and $T_{2}$ in $S_{1}$ and $S_{2}$. The temperatures are given by

$$
\begin{aligned}
\overline{\boldsymbol{T}} & =\left(\begin{array}{c}
T_{1} \\
T_{2}
\end{array}\right)=\frac{1}{2 \pi k}\left(\begin{array}{cc}
\frac{1}{\sqrt{(x-a)^{2}+y^{2}}} & \frac{1}{\sqrt{(x+a)^{2}+y^{2}}} \\
\frac{1}{\sqrt{\left(x^{\prime}-a\right)^{2}+y^{\prime 2}}} & \frac{1}{\sqrt{\left(x^{\prime}+a\right)^{2}+y^{\prime 2}}}
\end{array}\right) \\
& \times\left(\begin{array}{c}
P_{1} \\
P_{2}
\end{array}\right)=\overline{\overline{\boldsymbol{A}}}\left(\begin{array}{c}
P_{1} \\
P_{2}
\end{array}\right)=\overline{\overline{\boldsymbol{A}}} \overline{\boldsymbol{P}} .
\end{aligned}
$$

We are only interested to investigate the existence or the uniqueness of the solution of the inverse problem. In other words is it possible to calculate the inverse matrix $\overline{\overline{\boldsymbol{A}}}^{-1}$. Therefore the equation

$$
\operatorname{det} \overline{\overline{\boldsymbol{A}}}=0
$$

has to be considered first. After some calculations Eq. (4) gives rise to

$$
\left(x-x_{0}\right)^{2}+y^{2}=x_{0}^{2}-a^{2}=R^{2},
$$

where

$$
x_{0}=\frac{x^{\prime 2}+y^{\prime 2}+a^{2}}{2 x^{\prime}}
$$

is the equation of a circle with radius $R=\sqrt{x_{0}^{2}-a^{2}}$, centered around the point $\left(x_{0}, 0\right)$. In classical geometry, this is known as the circle of Appolonius. An illustrative example is drawn in Fig. 1. If $x^{\prime}=-2 a$ and $y^{\prime}=0$, one obtains $x_{0}=-5 / 4 a$ and $R=3 / 4 a$. If the second sensor point $(x, y)$ is located on this circle, the inverse thermal problem cannot be solved. These results can be understood physically. For any point of the Appolonius circle, the ratio $d_{1} / d_{2}$ is constant and the same ratio of temperatures $T_{1} / T_{2}$ will be sensed. Remark that the circle is not an isothermal line.

If $x^{\prime}=y^{\prime}=0$ the Appolonius circle coincides with the $y$-axis. The second sensor cannot help us to decide whether a heat source is located in $P_{1}$ or $P_{2}$. Interchanging the two heat sources will give the same temperatures at the sensing points.

The circle of Appolonius tells us where the determinant is exactly zero and hence the inverse problem cannot be solved at all. In practice one is dealing with ill conditioning. It has been pointed out by several authors that the value of the determinant is not a good criterion for the ill conditioning of the matrix [15]. The condition number defined as the quotient of the biggest eigenvalue and the smallest one is used to measure the ill conditioning of a matrix. However, it was proved that it is much better to use the so-called singular values instead of the eigenvalues. In order to calculate the singular values, the matrix $\overline{\overline{\boldsymbol{A}}}$ has to be multiplied by its Hermitian conjugate $\overline{\overline{\boldsymbol{A}}}^{\mathrm{H}}$ :

$$
\overline{\overline{\boldsymbol{A}}}^{\mathrm{H}} \overline{\overline{\boldsymbol{A}}} \text {. }
$$

$\overline{\overline{\boldsymbol{A}}}^{\mathrm{H}}$ is the transpose of $\overline{\overline{\boldsymbol{A}}}$ whereas all elements have been replaced by their complex conjugates. The eigenvalues $\lambda_{i}$ of $\overline{\overline{\boldsymbol{A}}} \mathrm{H} \overline{\overline{\boldsymbol{A}}}$ are calculated. It has been proved that all these eigenvalues are real and positive. The singular values $\sigma_{i}$ are then found as

$$
\sigma_{i}=\sqrt{\lambda_{i}}
$$

The condition number $\kappa$ is then defined as:

$$
\kappa=\frac{\sigma_{\max }}{\sigma_{\min }},
$$

where $\sigma_{\max }$ and $\sigma_{\min }$ denote the maximum and the minimum singular values. The closer the condition number $\kappa$ is to 1 the better the matrix $\overline{\overline{\boldsymbol{A}}}$ will be conditioned.

The use of singular values has the advantage that $\overline{\overline{\boldsymbol{A}}}^{\mathrm{H}} \overline{\overline{\boldsymbol{A}}}$ is always a square $n \times n$ matrix even when $\overline{\overline{\boldsymbol{A}}}$ is a rectangular one. This situation often occurs in practical situations when more sensors than heat sources are involved.

The following case with two heat sources and two sensors has been analysed using the singular value approach. 


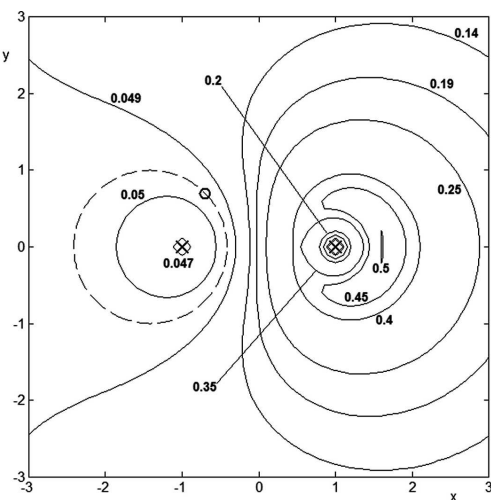

Fig. 2. Two heat sources and two sensors case. Map of the inverse condition number as a function of the position of the second sensor.

The two heat sources are placed in the points $(-1,0)$ and $(+1,0)$ as shown in Fig. 2. One sensor $S_{1}$ is in a fixed position $(-0.7,0.7)$. The other sensor $S_{2}$ could be moved in the area $-3<x<+3$ and $-3<y<+3$. For each position $(x, y)$ of the second sensor $S_{2}$ the condition number $\kappa$ of the matrix $\overline{\bar{A}}$ was evaluated numerically. The results are plotted in Fig. 2. Instead of plotting $\kappa$, the inverse condition number $\kappa^{-1}$ has been plotted to avoid $\kappa=\infty$ which happens when one of the singular values turns out to be zero. The circle of Appolonius is marked with the dashed line. The optimal position of the second sensor, i.e. the position such that the condition number is as close as possible to unity, is at the point $(1.6,0)$ where $\kappa^{-1}=0.5$ or $\kappa=2$. Intuitively, one should expect that the optimal position of the second sensor should be very near to one of the heat sources. It will be proved mathematically later on in Sect. 5 that the optimal position is not necessarily in the near neighbourhood of the heat source. The circle of Appolonius has also been drawn in Fig. 2. As expected, the condition number $\kappa$ becomes very high if the second sensor approaches the circle of Appolonius.

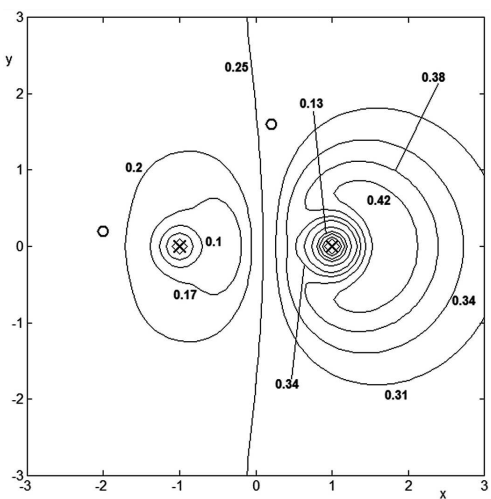

Fig. 3. Two heat sources and three sensors case. Map of the inverse condition number as a function of the position of the third sensor.
A second example involving two heat sources and three sensors is shown in Fig. 3. The heat sources are again in the positions $(-1,0)$ and $(+1,0)$. Two sensors are at fixed positions $(0.2,1.6)$ and $(-2,0.2)$, the third sensor has variable coordinates $(x, y)$. Again one observes that the condition number gets worse if the third sensor is approaching one of the heat sources. The optimal position of the third sensor is found to be at $(1.8,0)$ with a corresponding value of $\kappa^{-1}=0.45$ or a condition number $\kappa=2.22$.

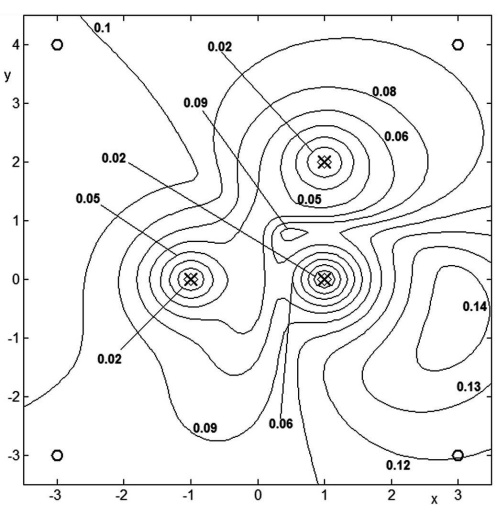

Fig. 4. Three heat sources and five sensors case. Map of the inverse condition number as a function of the position of the fifth sensor.

Figure 4 shows a simulation with three heat sources and five sensors. The heat sources are at the positions $(-1,0)(1,0)(1,2)$. Four sensors are fixed at the corners $(-3,4),(3,4),(3,-3)$ and $(-3,-3)$. This case corresponds to the actual situation of an integrated circuit where the sensors can only be placed on the boundary of the chip. The layout of the circuit is such that no free space exists in the middle of the chip. The map of $\kappa^{-1}$ due to the positions of the fifth sensor is shown in Fig. 4. It turns out that the optimal position is in the point $(3.1,-0.4)$ which is also on the rectangular boundary of the rectangle formed by the fixed sensors. The optimal value is $\kappa^{-1}=0.15$ or $\kappa=6.66$. Again the neighbourhood of the heat sources must be avoided which is an advantage from design point of view.

The dimensions $x$ and $y$ in Figs. $2-4$ are given in dimensionless form. The results (i.e. the condition number evaluation) do not vary if all dimensions are multiplied by a constant value. As already pointed out in the previous section, the actual distances between the sensors and heat sources should be less than or comparable to the substrate thickness due to the limited validity of the approximation (1).

\section{Practical placement of a sensor array around a circuit}

In this section we attack a practical problem: a circuit has been designed and there is no free space available to 
add temperature sensors and the related circuitry necessary for temperature monitoring. The only remaining possibility is to put the array of sensors along the boundary, so that a new and expensive integrated circuit design can be avoided.

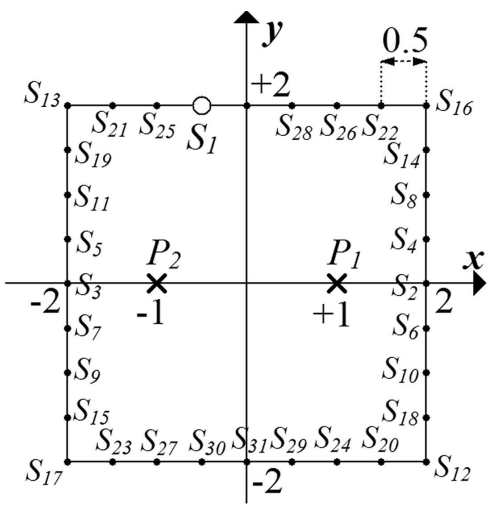

Fig. 5. Chip layout with two heat sources and up to 32 sensors on the boundary.

We consider again the situation involving two heat sources positioned in $(-1,0)$ and $(+1,0)$ as shown in Fig. 5. In contrast to the previous examples the sensors are only allowed to be placed on the circuit boundary which is a square with a side length of 4 units. Sensors can only be positioned in points with coordinates $x=n / 2$ and $y=m / 2$ where $n$ and $m$ are integer numbers in the interval $(-5,+5)$.

The iteration procedure is as follows: the first sensor $S_{1}$ is fixed in the point $(-0.5,2)$. A second sensor $S_{2}$ is introduced and the map of the condition number $\kappa$ is evaluated as outlined above. The sensor $S_{2}$ is then placed in the boundary point corresponding to the best condition number, which turns out to be the point $(2,0)$ and this position remains fixed during the remaining iteration procedure. The third sensor $S_{3}$ is introduced and its position is determined by searching the optimal condition number for all the free boundary points. This procedure is repeated till all the 32 boundary points are occupied, as shown in Fig. 5.

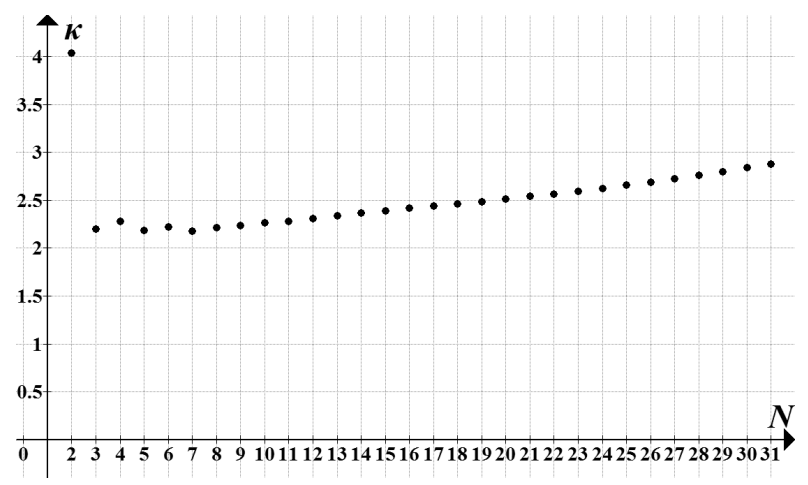

Fig. 6. Condition number as a function of the number $N$ of sensors.
Figure 6 shows the condition number $\kappa$ as a function of the number $N$ of sensors which are already placed. One observes that after some iteration steps the condition number increases linearly but still for the last step of the iteration procedure the condition number is around $\kappa=2$ which is a quite good value for a stable matrix inversion. Remark also that the increase of the condition number as a function of $N$ is rather small. Hence a large number of sensors is not a problem for the ill conditioning of the inverse problem.

Again we have here a case where all sensors are relatively far away from the heat sources and still the condition number is quite good so that the ill conditioning will not give rise to any numerical problems.

Putting the sensors one by one is not a guarantee that the optimal layout will be obtained. The results in Fig. 6 clearly show that for $N>5$ the condition number tends to stabilise. Furthermore it was found that other configurations did not have a major influence on the condition number as long as $N>5$. For small values of $N$ (e.g. $N=2$ ) the influence might be important.

\section{Theoretical analysis}

In order to explain the problem why the situation where one of the sensors is very close to a heat source is far from being optimal, two simple problems will be analysed here. Both can be calculated analytically so that a physical interpretation is straightforward.

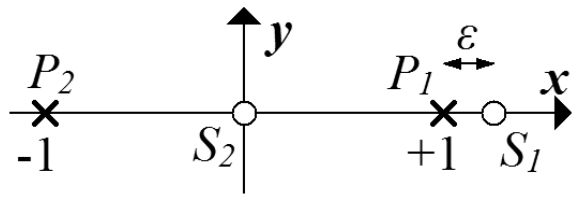

Fig. 7. Two heat sources and two-sensors case. One sensor $S_{1}$ is very close to the heat source $P_{1}$.

We consider the case of two heat sources and two sensors all positioned on the $x$-axis (Fig. 7 ). One fixed sensor $S_{2}$ is just halfway the two heat sources $P_{1}$ and $P_{2}$. The other sensor $S_{1}$ is at a very small distance $\varepsilon$ from the heat source $P_{1}$. The matrix $\overline{\overline{\boldsymbol{A}}}$ is then

$$
\overline{\overline{\boldsymbol{A}}}=\left(\begin{array}{cc}
\frac{1}{\varepsilon} & \frac{1}{2+\varepsilon} \\
1 & 1
\end{array}\right)
$$

and hence

$$
\overline{\overline{\boldsymbol{A}}}^{\mathrm{H}} \overline{\overline{\boldsymbol{A}}}=\left(\begin{array}{cc}
\frac{1}{\varepsilon^{2}}+1 & \frac{1}{\varepsilon(2+\varepsilon)}+1 \\
\frac{1}{\varepsilon(2+\varepsilon)}+1 & \frac{1}{(2+\varepsilon)^{2}}+1
\end{array}\right) .
$$

The eigenvalues $\lambda_{1}$ and $\lambda_{2}$ of (11) are found to be

$$
\begin{aligned}
& \lambda_{1}=\frac{1}{2 \varepsilon^{2}}\left(1+\sqrt{1-4 \varepsilon^{2}}\right) \approx \frac{1}{\varepsilon^{2}}, \\
& \lambda_{2}=\frac{1}{2 \varepsilon^{2}}\left(1-\sqrt{1-4 \varepsilon^{2}}\right) \approx 1 .
\end{aligned}
$$

The approximations (12) and (13) are valid for $\varepsilon \rightarrow 0$, 
i.e. the sensor $S_{1}$ is very close to the heat source $P_{1}$. Using the approximate values the condition number is then obviously

$$
\kappa=\frac{\sigma_{\max }}{\sigma_{\min }}=\sqrt{\frac{\lambda_{\max }}{\lambda_{\min }}}=\varepsilon,
$$

which means that for $\varepsilon \rightarrow 0$, the condition number $\kappa \rightarrow 0$ and the matrix $\overline{\overline{\boldsymbol{A}}}$ becomes highly ill conditioned.

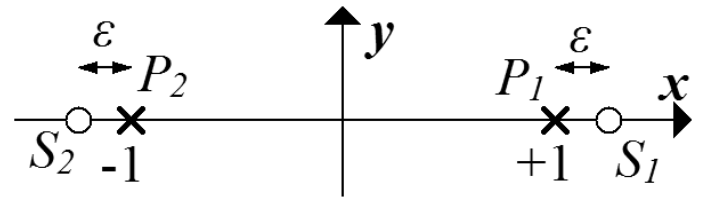

Fig. 8. Two heat sources and two-sensors case. Both sensors are very close to the heat sources.

Intuitively, one should expect that putting a sensor very close to a heat source should be the best situation to avoid ill conditioning. The numerical examples of the foregoing sections proved the opposite. Therefore a second problem shown in Fig. 8 is analysed now in detail.

The matrix is now

$$
\overline{\overline{\boldsymbol{A}}}=\left(\begin{array}{cc}
\frac{1}{\varepsilon} & \frac{1}{2+\varepsilon} \\
\frac{1}{2+\varepsilon} & \frac{1}{\varepsilon}
\end{array}\right)
$$

and

$$
\overline{\overline{\boldsymbol{A}}} \mathrm{H} \overline{\overline{\boldsymbol{A}}}=\left(\begin{array}{cc}
\frac{1}{\varepsilon^{2}}+\frac{1}{(2+\varepsilon)^{2}} & \frac{2}{\varepsilon(2+\varepsilon)} \\
\frac{2}{\varepsilon(2+\varepsilon)} & \frac{1}{\varepsilon^{2}}+\frac{1}{(2+\varepsilon)^{2}}
\end{array}\right) .
$$

The eigenvalues of (16) are easily found to be

$$
\begin{aligned}
& \lambda_{1}=\left(\frac{1}{2+\varepsilon}-\frac{1}{\varepsilon}\right)^{2}, \\
& \lambda_{2}=\left(\frac{1}{2+\varepsilon}+\frac{1}{\varepsilon}\right)^{2} .
\end{aligned}
$$

The condition number is then

$$
\kappa=\frac{\sigma_{\max }}{\sigma_{\min }}=\frac{\frac{1}{2+\varepsilon}+\frac{1}{\varepsilon}}{\frac{1}{2+\varepsilon}-\frac{1}{\varepsilon}} \approx\left(1+\frac{\varepsilon}{2}\right)\left(1-\frac{\varepsilon}{2}\right) \approx 1 .
$$

For small values of $\varepsilon$, i.e. both sensors are very near a heat source, the condition number $\kappa \rightarrow 1$ which is the best possible situation from numerical point of view.

\section{Conclusion}

In this paper we considered the inverse heat problem on a semiconductor chip. This problem has gained attention because real time monitoring of the temperature distribution might be necessary to improve the life time of integrated circuits. The temperature sensors have to detect that all the transistors or subcircuits have the normal power dissipation.

It has been proved that the inverse heat problem can give rise to mathematical instabilities. Several configurations were treated numerically. It was found that placing a sensor near a heat source gives rise to a worse condition number. If all sensors are at comparable distances away from the heat sources, the condition number is found to be quite good. This result was checked with the help of a very simple case where a complete analytical solution was possible. The numerical results were confirmed. It was also found that if all sensors are very close to the heat sources, the condition number is also quite good. However this situation cannot be realised in practical layouts of integrated circuits without difficulties.

\section{Acknowledgments}

B. Bogusławski wants to thank the EU for finantial support for his stay at the University of Ghent within the framework of the Erasmus student exchange programme.

\section{References}

[1] K. Azar, J. Electron. Cool. 6, 42 (2000).

[2] G. De Mey, in: Proc. Thermal Problems in Electronics - Advanced Training Course Mixed Design of VLSI Circuits Proc., Dębe (Poland), Eds: A. Napieralski, Z. Ciota, A. Martinez, G. De Mey, J. Cabestany, Kluwer Academic Publishers, Boston 1994, p. 25.

[3] J.E. Solomon, IEEE J. Solid State Circ. 9, 314 (1974).

[4] A.L. Cavaglia, A.A. Iliadis, IEEE Electron Dev. Lett. 14, 133 (1993).

[5] M. Janicki, J. Soraghan, A. Napieralski, in: Proc. Conf. on Mixed Design of Integrated Circuits and Systems (MIXDES), Łódź (Poland), Ed: A. Napieralski, DMCS, Technical University of Łódź, Łódź 1998, p. 209.

[6] S. Kindermann, M. Janicki, Electron. Trans. Numer. Anal. 35, 164 (2009).

[7] M. Janicki, S. Kindermann, Inverse Problems Sci. Eng. 17, 1129 (2009).

[8] G. Stoltz, ASME J. Heat Transfer 82, 20 (1960).

[9] J. Beck, ASME J. Heat Transfer 62, 46 (1962).

[10] J. Beck, B. Blackwell, A. Haji-Sheikh, Int. J. Heat Mass Transfer 39, 3649 (1996).

[11] O. Alifanov, Inverse Heat Transfer Problems, Springer, New York 1994.

[12] V. Székely, M. Tencz, S. Török, G. Farkas, Cs. Márta, in: Proc. Advances in the Thermal Testing of IC's 3rd Int. Workshop on Thermal Investigation of IC's and Microstructures (THERMINIC), Cannes, Ed: B. Courtois, CMP Laboratory, Grenoble 1997, p. 5 .

[13] D.J. Dean, Thermal Design of Electronic Circuit Boards and Packages, Electrochemical Publications, Ayr, Scotland 1985.

[14] N. Rinaldi, Solid State Electron. 44, 1789 (2000).

[15] G. Golub, C. Van Loan, Matrix Computations, The John Hopkins University Press, Baltimore 1996. 